Nephrologe 2013 · 8:7-9

DOI 10.1007/s11560-012-0706-z

Online publiziert: 6. Januar 2013

(c) Springer-Verlag Berlin Heidelberg 2013

L.C. Rump ${ }^{1}$ D. Fliser ${ }^{2} \cdot$ H. Haller ${ }^{3}$

${ }^{1}$ Klinik für Nephrologie, Universitätsklinikum Düsseldorf

${ }^{2}$ Klinik für Innere Medizin IV, Nieren- und Hochdruckkrankheiten,

Universitätsklinikum des Saarlandes, Homburg/Saar

${ }^{3}$ Klinik für Nieren- und Hochdruckerkrankungen, Medizinische Hochschule Hannover

\title{
Herausgeberwechsel in Der Nephrologe
}

kenhauses Neuss sprach sich die Medizinische Fakultät der Heinrich-Heine-Universität 1983 dafür aus, eine eigenständige Abteilung für Nephrologie zu gründen. Seit dieser Zeit hat Prof. Grabensee die Klinik für Nephrologie in seiner gesamten Breite vertreten und weiterentwickelt. Besonders hervorzuheben ist sein Engagement in der Intensivmedizin, das auch darin zum Ausdruck kommt, dass die Klinik für Nephrologie die Notaufnahmestation für das gesamte Zentrum für Innere Medizin und Neurologie leitet.

Ein weiterer Schwerpunkt von Prof. Grabensee ist der Bereich der Transplantationsmedizin. Hier hat er gemeinsam mit der Klinik für Gefäßchirurgie unter der Leitung von Prof. Sandmann ein ideales Modell einer interdisziplinären Zusammenarbeit auf dem Gebiet der Nierentransplantation etabliert. In diesem Düsseldorfer Konzept werden die Patienten unmittelbar vor und nach der Nierentransplantation von der Klinik für Nephrologie betreut, stets in sehr enger Kooperation mit den Operateuren. Mit diesem interdisziplinären Konzept wurden unter der Leitung von Prof. Grabensee mehr als 2000 Nierentransplantationen durchgeführt. Weitere klinische Schwerpunkte stellen die Diagnostik und die Therapie von Patienten mit immunologischen Systemerkrankungen mit Nierenbeteiligung dar. Alle Dialyseverfahren wurden hier von Prof. Grabensee frühzeitig etabliert, und auch die ambulante Versorgung wurde in Kooperation mit dem Kuratorium für Heimdialyse und den umliegenden nephrologischen Schwerpunkt- zentren in beispielhafter Weise durchgeführt.

Die Forschung von Prof. Grabensee war eng mit aktuellen klinischen Fragestellungen in Diagnostik und Therapie verbunden. Kardiovaskuläre Erkrankungen bei Patienten mit chronischer Niereninsuffizienz, die Progression chronischer Glomerulonephritiden und Untersuchungen zur Funktion der Niere nach Transplantation einschließlich des Einsatzes neuer immunsuppressiver Regime zählten zu den Schwerpunkten der Klinik. Die Peritonealdialyse war und ist ein besonderes Steckenpferd von Prof. Grabensee.

Prof. Grabensee war nicht nur Präsident der Gesellschaft für Nephrologie mit Jahreskongressen in Düsseldorf 1994, 1998 und 2002, sondern auch Präsident der Gesellschaft für Internistische Intensivmedizin und Notfallmedizin. Er ist Autor von mehr als 450 nationalen und internationalen Publikationen. Seit 1984 hat er $17 \mathrm{Ha}$ bilitationen und mehr als 80 Promotionen betreut. Er war Mitglied zahlreicher Editorial Boards, DFG-Gutachter und Mitglied in Preiskomitees sowie mehrfach gewähltes auswärtiges Kommissionsmitglied bei der Besetzung von Lehrstühlen an deutschen Universitäten.

Eine vollständige Liste aller Leistungen von Prof. Grabensee im nephrologischen Fachgebiet aufzulisten, würde den Rahmen dieses Vorworts sprengen. Seine besondere Stärke ist die Zuwendung zu Patienten, aber auch zu Mitarbeitern. Ihn haben immer die Vielfältigkeit von $\mathrm{Pa}$ tientenversorgung, Ausbildung von jungen Ärzten und Studierenden sowie die wissenschaftlichen Tätigkeiten in einem
Posten als Direktor der gesamten Medizinischen Klinik des Städtischen Kran- 
Team interessiert. Seine offene rheinische Art und den unerschütterlichen Glaube an Kompromisslösungen haben viele Kollegen schätzen gelernt. Prof. Grabensee ist ein Mann des Ausgleichs, der von der Liebe zum Fach Nephrologie lebt. Die Klinik für Nephrologie, der er von 1984 bis 2007 vorstand, war ihm selbst seine wichtigste Verpflichtung. Diese Klinik hat er mit Fleiß, Können und weiser Voraussicht zu voller Blüte gebracht, und sie wurde bis zu seiner Emeritierung in Forschung, Lehre und Patientenversorgung zu einem unverzichtbaren Bestandteil der Medizinischen Fakultät und des Universitätsklinikums. So spiegeln in gewisser Weise der klinische und der wissenschaftliche Lebenslauf von Prof. Grabensee die positive Entwicklung des Fachs Nephrologie in Deutschland wider. Das Lebenswerk von Prof. Grabensee an der Heinrich-HeineUniversität kann mit Fug und Recht beispielhaft genannt werden und für andere Standorte universitärer Nephrologien als Vorbild dienen.

Es war klar, dass Prof. Grabensee sich bei der bekannten körperlichen Fitness nach seiner Emeritierung 2007 nicht zur Ruhe setzen würde, sondern weiter klinisch, aber vor allen Dingen auch akademisch für die Gesellschaft der Nephrologie tätig sein würde. In seiner Zeit als Geschäftsführer der Akademie Niere seit 2008 hat er 88 Veranstaltungen organisiert, die Schirmherrschaft bei Fremdveranstaltungen mit definierten Voraussetzungen übernommen, Fremdveranstaltungen zertifiziert und CME-Zertifikate beantragt. Wichtig war ihm immer die Kooperation mit dem Verband Deutsche Nierenzentren und dem KfH.

Nicht zuletzt ist Prof. Grabensee als Mensch, Arzt und Hochschullehrer eine von Patienten und Kollegen hochgeschätzte Persönlichkeit. Für viele von uns ist er ein Vorbild an Fleiß, Kontinuität und Erfolg der akademischen Nephrologie. Ob privat oder bei offiziellen Anlässen stets ist es ein großes Vergnügen, ihn und seine Frau Antje zu treffen und angeregte Gespräche zu führen. Für seinen weiteren Lebensweg wünschen wir ihm alles Gute, Gesundheit und etwas mehr Zeit für sich und die Familie nach Abgabe der Aufgaben der Akademie.

\section{Zur Begrüßung von Herrn Prof. Dr. Gert Mayer}

Im Kreise der Herausgeber möchten wir ganz herzlich Herrn Prof. Dr. Gert Mayer begrüßen, Direktor der Universitätsklinik für Innere Medizin IV - Nephrologie und Hypertensiologie - im Landeskrankhaus Innsbruck. Für das Herausgeber-Board ist er in zweierlei Hinsicht eine wertwolle Ergänzung: Als klinisch und wissenschaftlich vielseitig tätiger Nephrologe ist er bestens mit aktuellen Themen des Fachs vertraut. Darüber hinaus ist Prof. Mayer als erster Herausgeber aus Österreich ein wichtiger Vermittler der Bedürfnisse der österreichischen Kollegen in puncto Fortund Weiterbildung.

Im Januar 2013

Für die Herausgeber

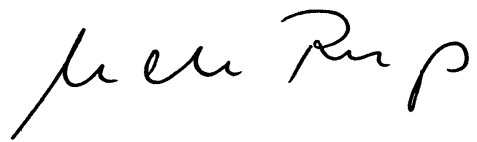

L.C. Rump

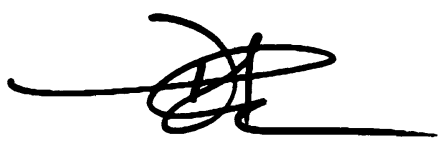

D. Fliser

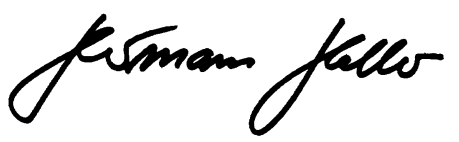

H. Haller

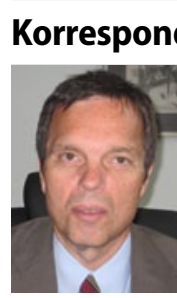

Prof. Dr. L.C. Rump

Klinik für Nephrologie, Universitätsklinikum Düsseldorf Moorenstraße 5 40225 Düsseldorf christian.rump@ med.uni-duesseldorf.de

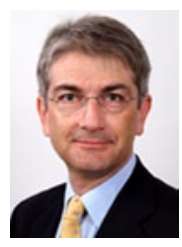

Prof. Dr. D. Fliser

Klinik für Innere Medizin IV, Nieren- und Hochdruckkrankheiten, Universitätsklinikum des Saarlandes Kirrberger Straße, 66421 Homburg/Saar danilo.fliser@ uniklinikum-saarland.de

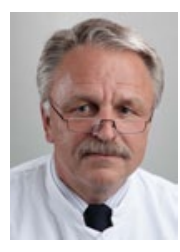

Prof. Dr. H. Haller

Klinik für Nieren- und Hochdruckerkrankungen, Medizinische Hochschule Hannover Carl-Neuberg-Str. 1, 30625 Hannover haller.hermann@ mh-hannover.de 
Hier steht eine Anzeige.

黛 Springer 